\title{
Synthesis of New Schiff Base: 4-[(Pyridin-3-ylmethylene)- amino]phenyldodecanoate
}

\author{
Sie-Tiong Ha ${ }^{1 *}$, Lay-Khoon Ong ${ }^{1}$, Yip-Foo Win ${ }^{1}$, Teck-Ming Koh ${ }^{1}$ and Guan-Yeow Yeap ${ }^{2}$ \\ ${ }^{1}$ Faculty of Engineering \& Science, Universiti Tunku Abdul Rahman, Jln Genting Kelang, 53300 \\ Kuala Lumpur, Setapak 53300 Kuala Lumpur, Malaysia. \\ ${ }^{2}$ Liquid Crystal Research Laboratory, School of Chemical Sciences, Universiti Sains Malaysia, \\ Minden 11800 Minden, Penang, Malaysia
}

* Author to whom correspondence should be addressed; E-mail: hast@mail.utar.edu.my

Received: 16 November 2008 / Accepted: 21 November 2008 / Published: 23 November 2008

\begin{abstract}
A new Schiff base 4-[(pyridin-3-ylmethylene)amino]phenyldodecanoate was synthesized via Steglich esterification. IR, ${ }^{1} \mathrm{H}$ NMR, ${ }^{13} \mathrm{C}$ NMR and MS spectroscopic data are presented.
\end{abstract}

Keywords: 4-[(Pyridin-3-ylmethylene)amino]phenyldodecanoate; Schiff base, alkyl chain.

$N$-benzylideneaniline Schiff bases have received a considerable amount of attention from many researchers owing to their importance in exhibiting thermochromism and photochromism [1]. In view of the importance of these compounds, chemists are prompted to generate the derivatives by introducing different substituents into the existing skeleton of the molecule. The presence of a long alkyl chain at the para position of the aldehyde and aniline fragments of Schiff bases has been regarded as one of the important elements which favours the existence of liquid crystal phases [2-5]. 


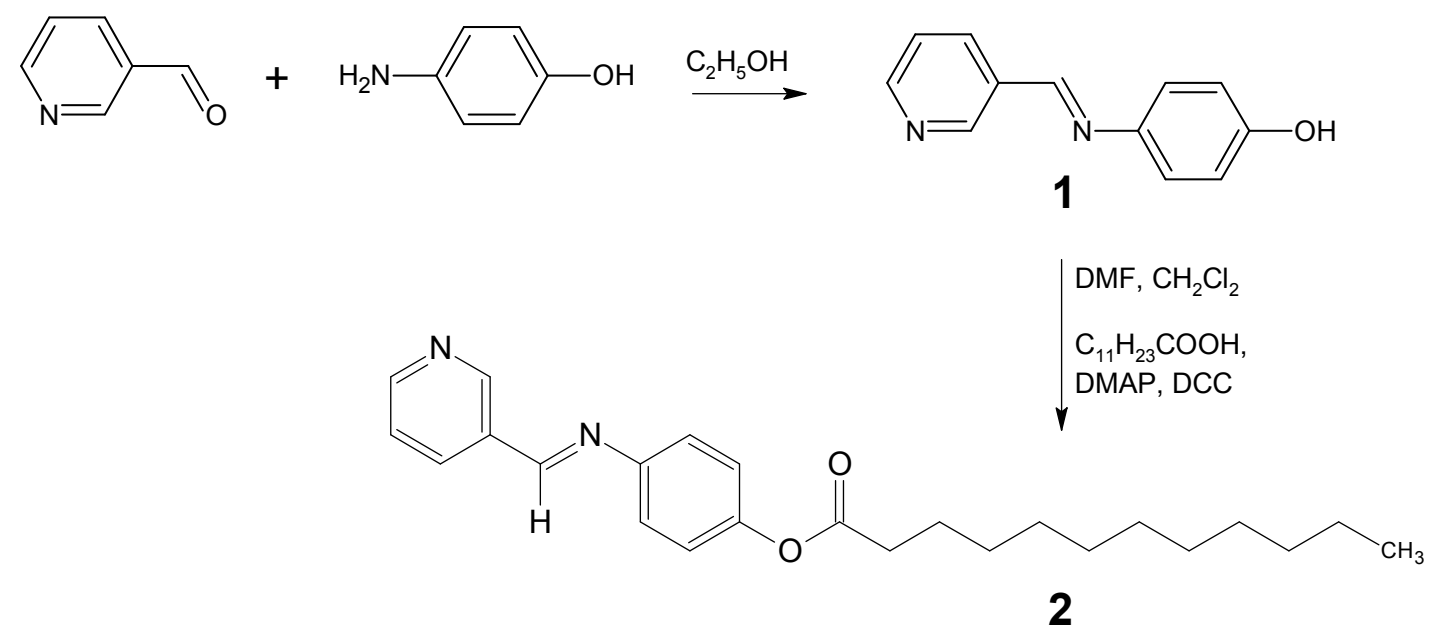

A solution of 3-pyridinecarbaldehyde (4.28 g, $40 \mathrm{mmol})$ and 4-aminophenol $(4.37 \mathrm{~g}, 40 \mathrm{mmol})$ in absolute ethanol $(70 \mathrm{~mL})$ was heated under reflux for 3 hours. Compound 1 thus obtained was recrystallized from absolute ethanol. Then, Schiff base 1 (3.96 g, $20 \mathrm{mmol}$ ) in dimethylformamide (DMF) (4 mL), was added to a solution of lauric acid (4.01 g, $20 \mathrm{mmol})$ and 4-dimethylaminopyridine (DMAP) $(1.22 \mathrm{~g}, 10 \mathrm{mmol})$ in dichloromethane $(70 \mathrm{~mL})$. The resulting mixture was stirred in an ice bath. To this solution, N,N'-dicyclohexylcarbodiimide (DCC) (4.12 g, $20 \mathrm{mmol})$ dissolved in dichloromethane $(20 \mathrm{~mL})$ was added dropwise while stirring in the ice bath for an hour. The resulting mixture was subsequently stirred at room temperature for another 3 hours. Then, the reaction mixture was filtered and the excess solvent was removed from the filtrate by evaporation. Recrystallization from absolute ethanol gave the Schiff base 2 as gray solid (2.21 g, 29\%).

Melting Point: $80.0^{\circ} \mathrm{C}$.

$\operatorname{MS}(\mathrm{EI}): \mathrm{M}^{+}(\mathrm{m} / \mathrm{z})=380$

IR (KBr, cm $\left.{ }^{-1}\right): 2953,2916,2849(\mathrm{C}-\mathrm{H}$ aliphatic); $1755(\mathrm{C}=\mathrm{O}$ ester $) ; 1626(\mathrm{C}=\mathrm{N}) ; 1595,1498$ $(\mathrm{C}=\mathrm{C}$ aromatic $)$.

${ }^{1} \mathrm{H}$ NMR (400 MHz, $\left.\mathrm{CDCl}_{3}\right): \delta / p p m 0.89\left(3 \mathrm{H}, \mathrm{t}, J=7.0 \mathrm{~Hz}, \mathrm{CH}_{3}\right), 1.29-1.44\left\{\mathrm{~m}, 16 \mathrm{H}, \mathrm{CH}_{3}\left(\mathrm{C}_{2}\right)_{8^{-}}\right.$ \}, 1.77 (qt, $2 \mathrm{H}, J=7.3 \mathrm{~Hz},-\underline{\mathrm{C}}_{2} \mathrm{CH}_{2} \mathrm{COO}$ ), 2.58 (t, $2 \mathrm{H}, J=7.5 \mathrm{~Hz},-\underline{\mathrm{C}}_{2} \mathrm{COO}$ ) $), 7.14$ (dd, $2 \mathrm{H}, J=$ $8.8 \mathrm{~Hz}, 2.2 \mathrm{~Hz}, \mathrm{Ar}-\mathrm{H}), 7.26$ (dd, 2H, $J=8.8 \mathrm{~Hz}, 2.2 \mathrm{~Hz}, \mathrm{Ar}-\mathrm{H}), 7.43$ (dd, $1 \mathrm{H}, J=7.8 \mathrm{~Hz}, 4.8 \mathrm{~Hz}$, Ar-H), $8.30(\mathrm{dd}, 1 \mathrm{H}, J=7.9,1.9 \mathrm{~Hz}, \mathrm{Ar}-\mathrm{H}), 8.51(\mathrm{~s}, 1 \mathrm{H}, \mathrm{CH}=\mathrm{N}), 8.70(\mathrm{dd}, 1 \mathrm{H}, J=4.7 \mathrm{~Hz}, 1.5 \mathrm{~Hz}$, Ar-H), 9.02 (d, 1H, $J=1.9 \mathrm{~Hz}, \mathrm{Ar}-\mathrm{H})$.

${ }^{13} \mathrm{C}$ NMR (100 MHz, $\left.\mathrm{CDCl}_{3}\right): \delta / p p m 172.8(\mathrm{COO}), 157.6(\mathrm{CH}=\mathrm{N}), 152.5,151.4,149.8,149.3$,

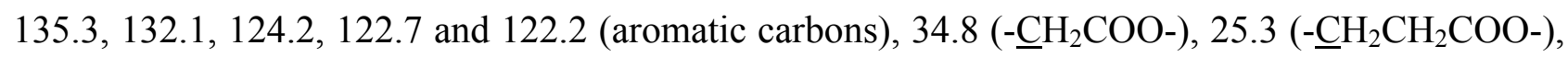
32.3, 30.0, 29.9, 29.7, 29.6, 29.5, 26.0 and $\left.23.1\left(\mathrm{CH}_{3}\left(\mathrm{C}_{2}\right)_{8}\right)^{-}\right), 14.5\left(\mathrm{CH}_{3}\right)$.

Elemental analysis: Calculated for $\mathrm{C}_{24} \mathrm{H}_{32} \mathrm{~N}_{2} \mathrm{O}_{2}: \mathrm{C}, 75.75 \%, \mathrm{H}, 8.48 \%, \mathrm{~N}, 7.36 \%$; Found: C, $75.70 \%, \mathrm{H}, 8.57 \%$, N, $7.41 \%$. 


\section{Acknowledgements}

The authors would like to thank Universiti Tunku Abdul Rahman and Universiti Sains Malaysia for financial support and research facilities.

\section{References}

1. Hadjoudis, E.; Vittorakis, M.; Moustakali-Mavridis, I. Tetrahedron 1987, 43, 1345-1360.

2. Yeap, G.Y.; Ha, S.T.; Lim, P.L.; Boey, P.L.; Mahmood, W.A.K.; Ito, M.M.; Sanehisa, S. Mol. Cryst. Liq. Cryst. 2004, 423, 73-84.

3. Yeap, G.Y.; Ha, S.T.; Lim, P.L.; Boey, P.L.; Ito, M.M.; Sanehisa, S.; Vill, V. Mol. Cryst. Liq. Cryst. 2006, 452, 63-72.

4. Yeap, G.Y.; Ha, S.T.; Boey, P.L.; Mahmood, W.A.K.; Ito, M.M.; Youhei, Y. Mol. Cryst. Liq. Cryst. 2006, 452, 73-90.

5. Yeap, G.Y.; Ha, S.T.; Lim, P.L.; Boey, P.L.; Ito, M.M.; Sanehisa, S.; Youhei, Y. Liq. Cryst. 2006, 33, 205-211.

(C) 2008 by the authors; licensee Molecular Diversity Preservation International, Basel, Switzerland. This article is an open-access article distributed under the terms and conditions of the Creative Commons Attribution license (http://creativecommons.org/licenses/by/3.0/). 